\title{
Immunogenicity of human embryonic stem cell-derived beta cells
}

\author{
Cornelis R. van der Torren ${ }^{1,2}$ • Arnaud Zaldumbide ${ }^{3}$ - Gaby Duinkerken ${ }^{1,2}$ • \\ Simone H. Brand-Schaaf ${ }^{1} \cdot$ Mark Peakman $^{4}$ • Geert Stangé ${ }^{2,5}$ • Laura Martinson ${ }^{6}$. \\ Evert Kroon $^{6} \cdot$ Eugene P. Brandon ${ }^{6}$ - Daniel Pipeleers ${ }^{2,5} \cdot$ Bart O. Roep $^{1,2,7}$
}

Received: 17 June 2016/Accepted: 14 September 2016 / Published online: 27 October 2016

(C) The Author(s) 2016. This article is published with open access at Springerlink.com

\begin{abstract}
Aims/hypothesis To overcome the donor shortage in the treatment of advanced type 1 diabetes by islet transplantation, human embryonic stem cells (hESCs) show great potential as an unlimited alternative source of beta cells. hESCs may have immune privileged properties and it is important to determine whether these properties are preserved in hESC-derived cells.

Methods We comprehensively investigated interactions of both innate and adaptive auto- and allo-immunity with hESC-derived pancreatic progenitor cells and hESC-derived endocrine cells, retrieved after in-vivo differentiation in capsules in the subcutis of mice.

Results We found that hESC-derived pancreatic endodermal cells expressed relatively low levels of HLA endorsing

Electronic supplementary material The online version of this article (doi:10.1007/s00125-016-4125-y) contains peer-reviewed but unedited supplementary material, which is available to authorised users.
\end{abstract}

\section{Bart O. Roep}

boroep@lumc.nl

1 Department of Immunohaematology and Blood Transfusion, E3-Q, Leiden University Medical Center, P.O. Box 9600, NL-2300 RC Leiden, the Netherlands

2 JDRF Center for Beta Cell Therapy in Diabetes, http://www.betacelltherapy.org/

3 Department of Molecular Cell Biology, Leiden University Medical Center, Leiden, the Netherlands

4 Department of Immunobiology, King's College London School of Medicine, London, UK

5 Diabetes Research Center, Brussels Free University-VUB, Brussels, Belgium

6 ViaCyte, Inc., San Diego, CA, USA

7 Department of Diabetes Immunology, Diabetes and Metabolism Research Institute at the Beckman Research Institute, City of Hope, Duarte, CA, USA protection from specific immune responses. HLA was upregulated when exposed to IFN $\gamma$, making these endocrine progenitor cells vulnerable to cytotoxic $\mathrm{T}$ cells and alloreactive antibodies. In vivo-differentiated endocrine cells were protected from complement, but expressed more HLA and were targets for alloreactive antibody-dependent cellular cytotoxicity and alloreactive cytotoxic T cells. After HLA compatibility was provided by transduction with HLAA2, preproinsulin-specific $\mathrm{T}$ cells killed insulin-producing cells.

Conclusions/interpretation hESC-derived pancreatic progenitors are hypoimmunogenic, while in vivo-differentiated endocrine cells represent mature targets for adaptive immune responses. Our data support the need for immune intervention in transplantation of hESC-derived pancreatic progenitors. Cell-impermeable macro-encapsulation may suffice.

Keywords Allograft rejection · Autoimmunity · Beta cells · Embryonic stem cells $\cdot$ Transplantation

$\begin{array}{ll}\text { Abbreviations } \\ \text { ADCC } & \text { Antibody-dependent cellular cytotoxicity } \\ \text { APC } & \text { Allophycocyanin } \\ \text { B-LCL } & \text { B lymphocytes } \\ \text { CMV } & \text { Cytomegalovirus } \\ \text { CTL } & \text { Cytotoxic T lymphocyte } \\ \text { DAF } & \text { Decay-accelerating factor } \\ \text { EBV } & \text { Epstein-Barr virus } \\ \text { EF1 } \alpha & \text { Elongation factor } 1 \alpha \\ \text { FITC } & \text { Fluorescein isothiocyanate } \\ \text { hESC } & \text { Human embryonic stem cell } \\ \text { hESC-EC } & \text { hESC-derived endocrine cell } \\ \text { hESC-PE } & \text { hESC-derived pancreatic endodermal cell } \\ \text { MCP } & \text { Membrane cofactor protein } \\ \text { MIP } & \text { Membrane attack complex-inhibitory protein }\end{array}$


NK Natural killer

PBMC Peripheral blood mononuclear cell

PPI Preproinsulin

PTEC Primary tubular epithelial cell

Th T helper

\section{Introduction}

Beta cell replacement by islet transplantation can functionally cure long-standing type 1 diabetes but its implementation is limited by the lack of donor organs, loss of graft function over time and the side effects of obligatory immunosuppression. Alternative sources of beta cells could overcome the shortage of human islets, while novel protective strategies and/or the potential for immune privilege of an alternative source of beta cells may help to overcome the immunosuppressive burden.

Several alternative sources of beta cells are currently being explored, including human embryonic stem cells (hESCs) [1], proliferating beta cell lines [2], induced pluripotent stem cells $[3,4]$ and xenogeneic islets [5, 6]. hESC-derived beta cells are well on the way to clinical translation with recent publications on improved safety and scaling of a protocol being made by Kroon and colleagues $[1,7,8]$.

After transplantation, hESC-derived beta cells face the challenges posed by the human immune system for the first time. Partial early loss of grafts through innate immune reactions may be overcome by transplanting more cells or by choosing an alternative site to the liver. However, both recurrent autoimmunity and alloreactive responses remain a persistent threat to transplanted human-derived beta cells despite immunosuppression [9-11]. Additionally, alloreactive responses provoked by a graft can be a risk for future transplantations [12-14]. Conversely, complete lack of immune interaction can leave cells vulnerable to infections or may invoke an innate immune attack by natural killer (NK) cells [15]. We recently showed this for immortalised beta cell lines [16].

Embryonic stem cells have immune privileged properties and can resist alloreactive responses [17]. However, differentiation of hESCs to other cell types can result in the loss of this immunological privilege [17-21]. Insights into the immunogenicity of the alternative sources of beta cells can help to guide the choice of immune-protective strategies and the relevant immune monitoring to be employed in clinical introduction. We therefore investigated the immunogenicity of hESCderived beta cells and their progenitors to adaptive immune responses relevant in transplantation.

\section{Methods}

Biological reagents hESC-derived pancreatic endodermal cells (hESC-PEs) were prepared from the CyT49 cell line
(HLA A1, A3, B44, B57) at ViaCyte (San Diego, CA, USA), cryopreserved and shipped to Brussels, as described [22]. For each series of experiments, cells were thawed and cultured for $72 \mathrm{~h}$ in db-N50-K50-E50 medium [7]. A fraction of the cells was sent to Leiden in $\mathrm{db}$ medium [1] and the remaining cells were transplanted in Brussels. Encapsulated grafts were prepared by loading $4 \times 10^{6}$ cells in a macro-device (ViaCyte) and were implanted in the subcutis of non-diabetic NOD/severe combined immunodeficiency (SCID) male mice (7-8 weeks old, NOD.CB17-Prkdcscid/J; Charles River, L'Arbresle, France). Implants were analysed in vivo and ex vivo as described [22]. In vivo graft function was followed over 20 weeks through measuring plasma human C-peptide levels 15 min after intraperitoneal injection of glucose; at posttransplant week 20, all recipients were confirmed to have reached plasma human C-peptide levels above $1 \mathrm{ng} / \mathrm{ml}$. Implants were resected at post-transplant week 20-25 and dispersed for analysis before sending to Leiden in phosphate-buffered Ham's F-10 medium (Gibco, Bleiswijk, the Netherlands), supplemented with $0.5 \%$ human albumin, $2 \mathrm{mmol} / 1 \mathrm{~L}-$ leucine and $2 \mathrm{mmol} / \mathrm{l}$ L-glutamine. Upon arrival, hESC-PEs and in vivo-differentiated cells were dissociated with $0.05 \%$ trypsin-EDTA $1 \mathrm{X}$ (Gibco) to single cells for immunological assays.

Human peripheral blood mononuclear cells (PBMCs) were separated from whole blood (for T cells) or buffy coats (for lymphocytes) by Ficoll-Hypaque density gradient. Peripheral blood lymphocytes were left over after CD14 depletion from PBMCs with CD14-MicroBeads according to the manufacturer's protocol (Miltenyi Biotec, Auburn, CA, USA). Preproinsulin (PPI)-specific T cell clone 1E6 generation was described previously [23]. Briefly, PBMCs from an individual with type 1 diabetes were stimulated with $\mathrm{PPI}_{15-24}$ peptide. PPI-specific cytotoxic T lymphocytes (CTLs) were sorted by FACS and expanded. Alloreactive CTL clones JS132 (to HLA-A2) and C776 (to HLA-A1) were generated by stimulating PBMCs, collected from a healthy donor, with irradiated Epstein-Barr virus (EBV)-transformed B cell line expressing the HLA of interest. After several rounds of stimulation and enrichment, the alloreactive population was cloned by limiting dilution at 0.5 cell/well [24]. CTL clones to cytomegalovirus (CMV) peptides VTEHDTLLY in HLA-A1 (clone 3c8) and NLVPMVATV in HLA-A2 (clone 18) were generated by single cell sorting of $\mathrm{CD}^{+}{ }^{+} \mathrm{T}$ cells stained with the respective tetramers and expanded using phytohaemagglutinin stimulation [25]. HEK-293 and primary tubular epithelial cells (PTECs, line HK-2) were cultured in DMEM/F-12 medium (Invitrogen, Landsmeer, the Netherlands) supplemented with $2 \mathrm{mmol} / \mathrm{l}$ L-glutamine, $25 \mathrm{mmol} / \mathrm{l} \mathrm{HEPES}, 50 \mathrm{U} / \mathrm{ml}$ penicillin and $50 \mu \mathrm{g} / \mathrm{ml}$ streptomycin (all purchased from Invitrogen) and, for PTECs, also $5 \mu \mathrm{g} / \mathrm{ml}$ insulin, $5 \mu \mathrm{g} / \mathrm{ml}$ transferrin, $5 \mathrm{ng} / \mathrm{ml}$ selenium, $36 \mathrm{ng} / \mathrm{ml}$ hydrocortisone and $10 \mathrm{ng} / \mathrm{ml}$ epidermal growth factor (all purchased from Sigma, Zwijndrecht, the Netherlands). B lymphocytes (B-LCL) lines were cultured 
in IMDM (Invitrogen) supplemented with $2 \mathrm{mmol} / 1$ L-glutamine, $25 \mathrm{mmol} / 1 \mathrm{HEPES}, 50 \mathrm{U} / \mathrm{ml}$ penicillin, $50 \mu \mathrm{g} / \mathrm{ml}$ streptomycin and $5 \% \mathrm{FBS}$.

Human monoclonal antibodies recognising HLA-A68 or HLA-B8 were selected from a panel as described previously [26]. In short, heterohybridomas were created by EBV transformation and cloning of B-LCL of multiparous women. The HLA specificities of the produced human monoclonal antibodies were validated using a complement-dependent cytotoxicity test on PBMCs.

Beta cell-specific $\mathrm{T}$ helper (Th) cell supernatant fractions were generated by incubating islet preparation reactive Th1 clone (1c6) from a diabetic patient with HLA-matched PBMCs pre-incubated with or without $10 \mu \mathrm{g} / \mathrm{ml}$ antigen in RPMI 1640 medium (Gibco) mixed without and with $11 \mathrm{mmol} / 1$ glucose to achieve $5.6 \mathrm{mmol} / \mathrm{l} \mathrm{D}$-glucose and supplemented with $2 \mathrm{mmol} / \mathrm{l}$ glutamine (Gibco) [27]. After 3 days the supernatant fraction was harvested and frozen until use.

For compatibility with autoreactive CTL clone 1E6, beta cell line EndoC- $\beta \mathrm{H} 1$ was transduced with a lentiviral vector containing HLA-A02:01 under elongation factor $1 \alpha(\mathrm{EF} 1 \alpha)$ promotor at multiplicity of infection of 2 . Third-generation self-inactivating lentivirus vectors were produced as described previously [28]. The generation of human cell lines and antibodies was carried out after obtaining informed consent and with approval of the institutional review board, in accordance with the 2008 revised principles of the Declaration of Helsinki.

\begin{abstract}
Alloreactivity assays Alloreactive cellular cytotoxicity was assessed by chromium release (PerkinElmer, Waltham, MA, USA). Briefly, dispersed hESC-derived cells were labelled with ${ }^{51} \mathrm{Cr}$ for $60 \mathrm{~min}$, washed three times and incubated with alloreactive $\mathrm{T}$ cells or, for antibody-dependent cellular cytotoxicity (ADCC), human monoclonal antibodies and peripheral blood lymphocytes in different effector-to-target ratios for 4-6 h or overnight. ${ }^{51} \mathrm{Cr}$ release in supernatant fractions was assessed on a WIZARD2 $\gamma$-counter (Perkin Elmer, Waltham, MA, USA). Specific lysis was calculated as [(experimental release - spontaneous release) / (max release - spontaneous release) $] \times 100 \%$.

Complement-dependent cytotoxicity was assessed with hESC-derived cells as targets in a clinical cross match assay [29]. In short, dispersed cells were incubated with serum containing alloreactive antibodies with known specificity and incubated in triplicate in Therasaki plates at room temperature for $1 \mathrm{~h}$. Rabbit complement (Inno-train, Frankfurt am Main, Germany) was added and the plates were incubated for another hour. Cell lysis was assessed by adding propidium iodideink solution and measured on the Patimed system (Leica, Rijswijk, the Netherlands). Minimal and maximum lysis was set to HLA-antibody-negative serum cell death and parallel lysis of HLA-matched lymphocyte targets, respectively.
\end{abstract}

Autoreactive assays At $20 \mathrm{~h}$ post transduction, virus supernatant fraction was removed from HLA-A02:01 transduced cells by centrifugation. The cells were cultured in Ham's F-10 medium (Gibco) supplemented with $200 \mathrm{mmol} / 1$ glutamine (Gibco), 0.5\% BSA fraction V (Sigma-Aldrich, Zwijndrecht, the Netherlands), $1 \mathrm{~mol} / 1 \mathrm{CaCl}_{2}$ and $1.8 \mathrm{~g} / \mathrm{l} \mathrm{D}$-glucose for 4 days to allow transgene expression before trypsin dispersion and incubation with PPI-specific CTL clone 1E6 or CMV-specific clone 18 in a 1:5 ratio. After overnight incubation, cells were stained with Fixable Viability Dye eFluor 450 (eBioscience, Vienna, Austria), CD45-PerCP (2D1; BD, Breda, the Netherlands) and HLA-A2-fluorescein isothiocyanate (FITC) (BD), then fixed in paraformaldehyde and permeabilised with saponin before staining with guinea pig anti-insulin (Free University Brussels, Brussels, Belgium) and donkey antiguinea pig Alexa 647 (Jackson Immunoresearch Laboratories, West Grove, PA, USA). Cells were measured on a CANTO II flow cytometer (BD). Transduction efficiency was determined by staining with anti-HLA-A2-Allophycocyanin (APC) (Bb7.2; BD) on a Calibur flow cytometer (BD).

Cell surface staining Cell surface antigens were analysed by FACS on a BD FACSCalibur after $20 \mathrm{~min}$ staining at $4^{\circ} \mathrm{C}$ with antibodies to HLA class I-FITC (W6/32; BD), HLA-DRFITC (L243; BD), IgG1-APC (MOPC-21; BD), IgG2aFITC (G155-178; BD), IgG2a-Phycoerythrin (G155-178; BD), anti-CD46-APC (MEM-258; ImmunoTools, Friesoythe, Germany), anti-CD55-Phycoerythrin (IA10; BD) and anti-CD59-APC (OV9A2, eBioscience).

\section{Experimental conditions}

Data were excluded if positive or negative controls failed. Additional intermediate titrations were performed in parallel in most samples that were left out for clarity to the reader. These data supported the conclusion. Cell lines for comparison were available in our laboratory unless stated otherwise and mycoplasma contamination was regularly excluded. Non-commercial antibodies had been generated and validated in our department before [26] and were of IgG subclass.

Statistical analysis Data are represented as mean and SE unless stated otherwise. GraphPad Prism 6.0 (GraphPad Software, La Jolla, CA, USA) was used to create graphs and perform analysis. Student's $t$ test was used to compare continuous data and Fisher's exact test was used for binominal data; $p<0.05$ was considered statistically significant. All immune assays were replicated three times. 

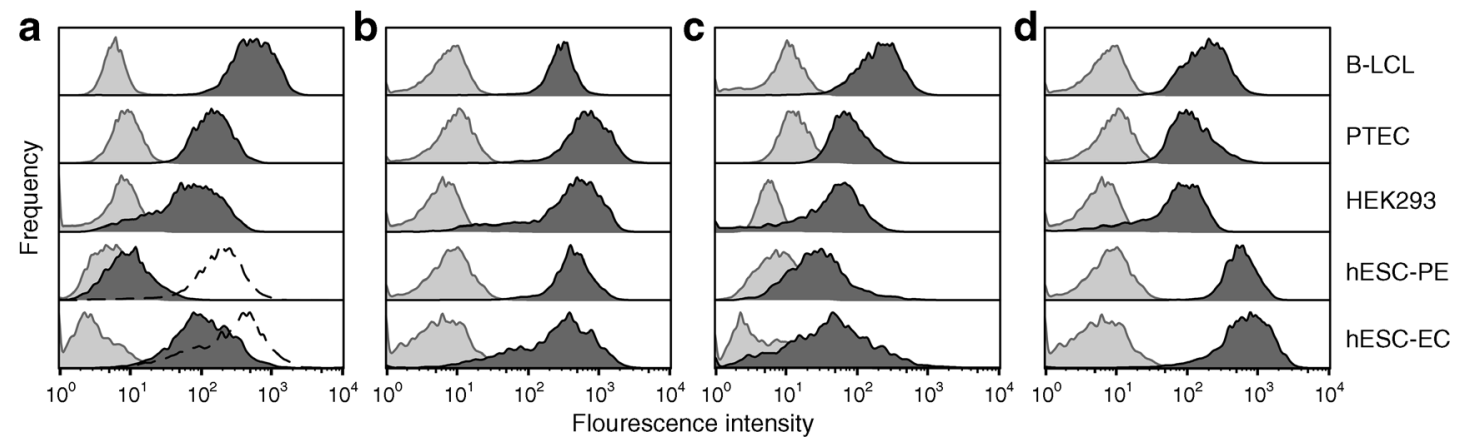

Fig. 1 Expression of HLA and complement receptors. FACS analysis of HLA class I (a) and complement inhibitory receptors CD46 (MCP) (b), CD55 (DAF) (c) and CD59 (MIP) (d) expression on trypsin-dissociated

hESC-PEs and differentiated hESC-ECs, compared with EBV-immortalised B-LCL, PTECs and HEK293 cells. HLA class I was upregulated by IFN $\gamma$ (1000 IU/ml; dashed line). Light traces represent isotype control staining

\section{Results}

Cell composition and function was assessed in hESC-PEs and cells retrieved from implants (hESC-derived endocrine cells [hESC-ECs]). These results and comparison with human islets have been reported [22]. Here, expression of HLA was assessed on the cell surface of hESC-PEs and hESC-ECs. This expression is essential for autoreactive, virus-specific $\mathrm{T}$ cell and alloreactive immune responses. hESC-PEs expressed very low levels of HLA class I, although HLA class I could be upregulated to reach levels expressed by other cell lines after exposure to the inflammatory cytokine, IFN $\gamma$ (Fig. 1a). On the other hand,

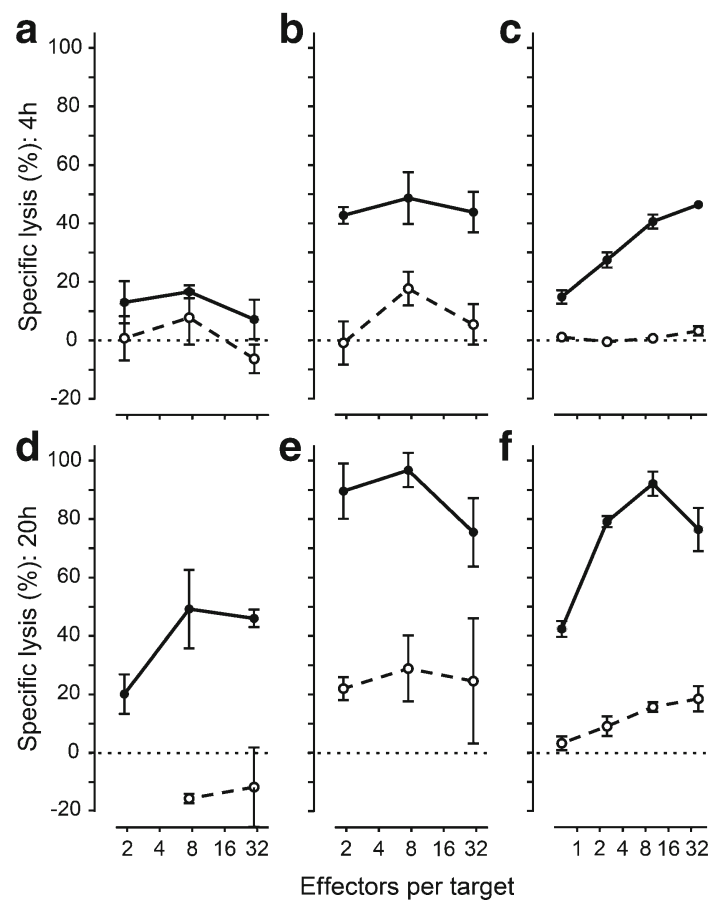

Fig. 2 Alloreactive and virus-specific CTLs can target hESC-PEs and differentiated hESC-ECs. ESC-PEs (a, b, d, e, $\mathbf{g}, \mathbf{h}, \mathbf{j}, \mathbf{k})$ and hESC-ECs $(\mathbf{c}, \mathbf{f}, \mathbf{i}, \mathbf{l})$ expressing HLA-A1 were labelled with ${ }^{51} \mathrm{Cr}$ and incubated with alloreactive CTLs targeting HLA-A1 (black circles, solid line) or targeting third party HLA-A2 (white circles, dashed line) $(\mathbf{a}-\mathbf{f})$ and virus-specific CTLs recognising CMV peptide in HLA-A1 on peptide-pulsed cells

the hESC-ECs expressed normal levels of HLA class I, which were only slightly upregulated by IFN $\gamma$. HLA class II is generally not expressed on endocrine cells. In line with this only minimal expression over isotype control was noted for hESCECs on $3.5 \%$ of cells (and on $7.7 \%$ of cells after upregulation with IFN $\gamma$ ). Also, expression of complement receptors, which protect cells from complement-mediated destruction, was assessed. Both hESC-PEs and hESC-ECs expressed membrane cofactor protein (MCP; CD46) at the level of other cell lines (Fig. 1b), together with high levels of membrane attack complex-inhibitory protein (MIP; CD59) (Fig. 1d). The differentiated hESC-ECs clearly expressed decay-accelerating factor

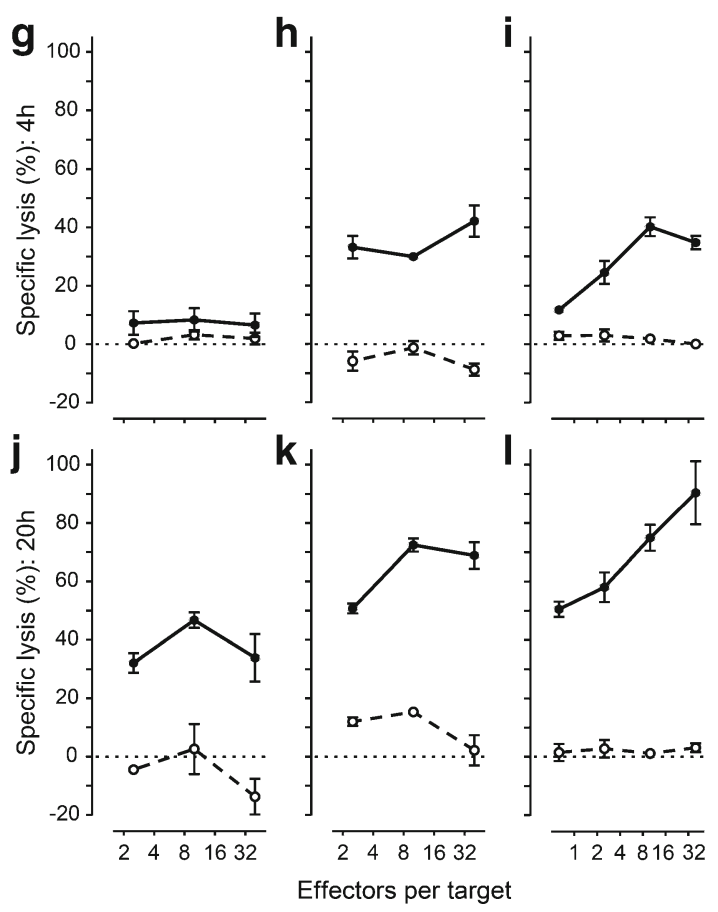

(black circles, solid line) or without peptide (white circles, dashed line) (g-l). Specific lysis after $4 \mathrm{~h}(\mathbf{a}-\mathbf{c}, \mathbf{g}-\mathbf{i})$ and $20 \mathrm{~h}(\mathbf{d}-\mathbf{f}, \mathbf{j}-\mathbf{l})$ was calculated relative to spontaneous lysis without $\mathrm{T}$ cells and chemically -induced maximum lysis. Inflammation was mimicked $(\mathbf{b}, \mathbf{e}, \mathbf{h}, \mathbf{k})$ by pre-incubation with IFN $\gamma(1000 \mathrm{IU} / \mathrm{ml})$, which upregulated HLA expression. Statistical results are available in ESM Fig. 1 
a

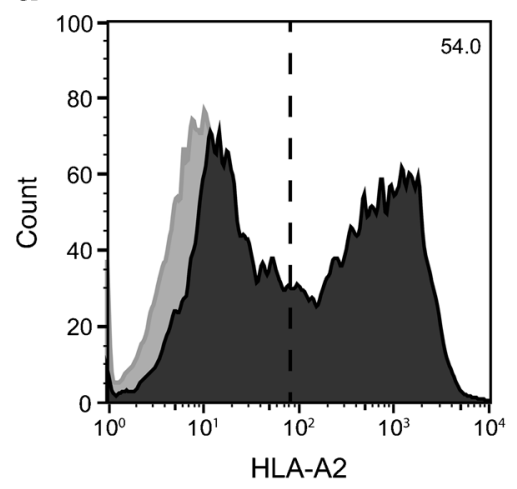

b
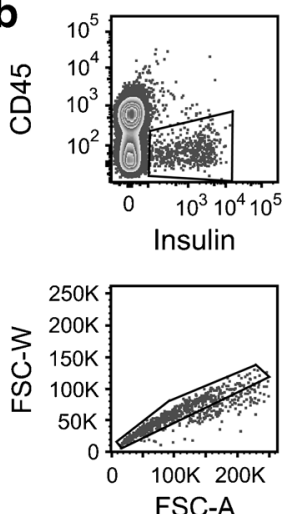

C

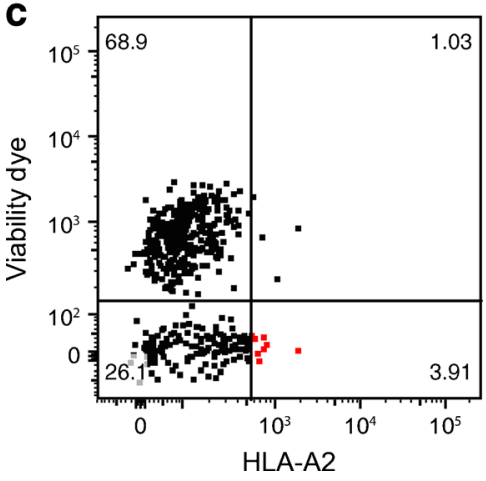

d

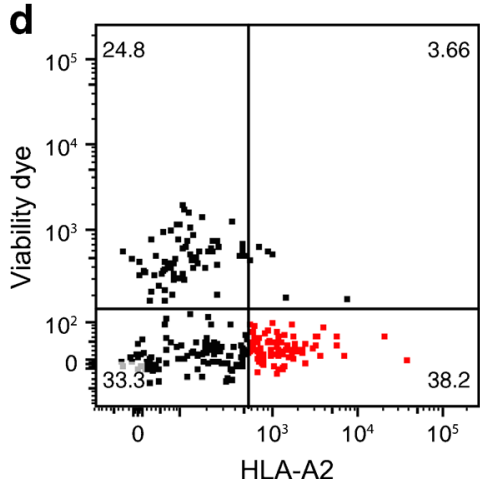

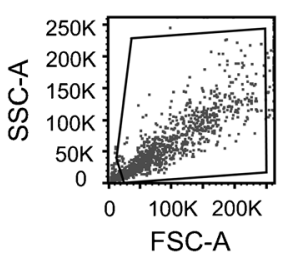

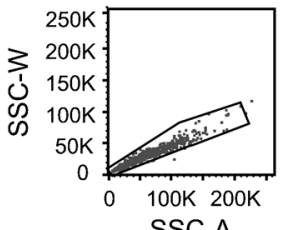

SSC-A

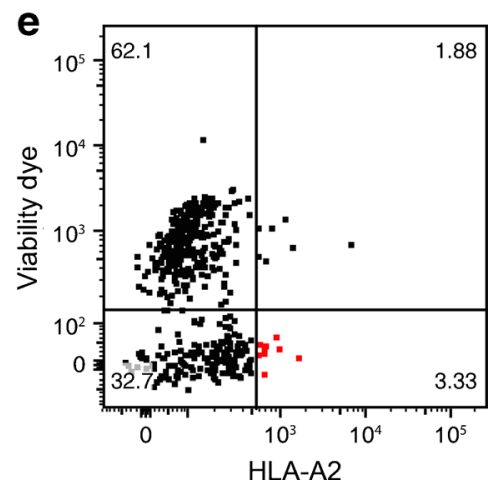

Fig. 3 PPI-specific CTLs recognise and kill hESC-derived beta cells. (a) hESC-ECs were transduced with HLA-A2 for compatibility with autoreactive CTL clone 1E6. FACS analysis of HLA-A2 expression showed expression on $>50 \%$ of hESC-ECs (dark grey; isotype control in light grey). (b) After 4 days' upregulation of the transgene, hESC-derived cells were incubated with autoreactive CTL clone 1E6 recognising PPI peptide in HLA-A2 or clone 18 recognising an irrelevant CMV peptide for $20 \mathrm{~h}$. Specific cytotoxicity was assessed by FACS by gating insulin-positive,

CD45-negative, single cells. (c, d) Gated beta cell-like cells expressing HLA-A2 (red) were killed overnight with $>90 \%$ efficiency by PPI-specific CTLs (c) compared with non-specific CTLs recognising CMV peptide (d). (e) The killing efficiency may be at maximum since this could not be increased by pulsing the beta cell-like cells with PPI peptide before exposure to PPI-specific CTLs. FSC-A, forward scatter: area; FSC-W, forward scatter: width; SSC-A, side scatter: area

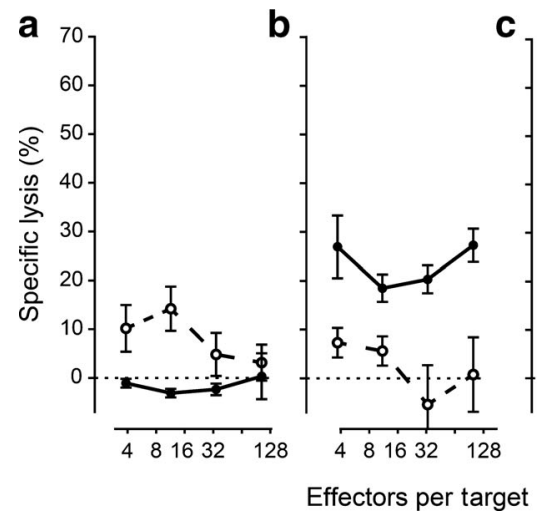

Fig. 4 Alloreactive antibodies induce cellular cytotoxicity but differentiated hESC-ECs resist complement-dependent cytotoxicity. (a-c) hESC-PEs (a, b) and differentiated hESC-ECs (c) were labelled with ${ }^{51} \mathrm{Cr}$ and incubated with specific (black circles, solid line) and non-specific (white circles, dashed line) alloreactive antibodies and peripheral blood lymphocytes for $6 \mathrm{~h}$. Chromium release was measured and specific lysis was calculated relative to spontaneous lysis without $\mathrm{T}$ cells and chemical induced maximum lysis. Inflammation was mimicked by pre-incubation with IFN $\gamma(1000 \mathrm{IU} / \mathrm{ml})$, upregulating HLA expression (b). (d, e) hESC-PEs (d) and hESC-ECs (e)

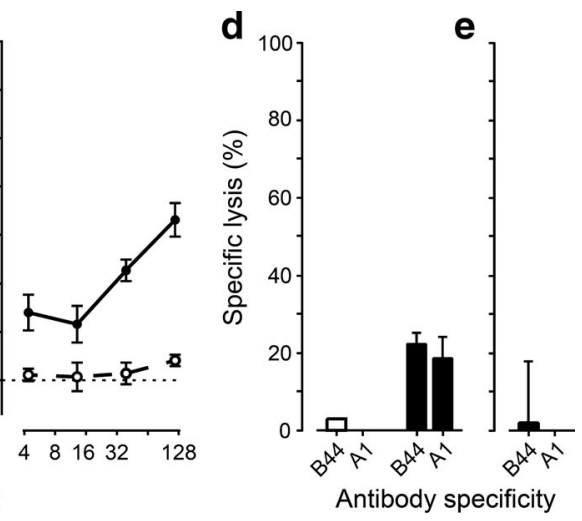

were incubated with serum containing HLA specific antibodies to A1 and B44 for $1 \mathrm{~h}$ and then for $1 \mathrm{~h}$ with rabbit complement. Lysis was assessed by propidium iodide staining with automated microscopy measurement and specific lysis calculated relative to cell death without specific antibodies and parallel specific lysis of lymphocyte controls. Inflammation was mimicked by pre-incubation with IFN $\gamma(1000 \mathrm{IU} / \mathrm{ml})$, upregulating HLA expression (black bars); white bars, no pre-incubation with IFN $\gamma$. hESC-ECs were only tested after IFN $\gamma$ pre-incubation. Statistical results are available in ESM Fig. 2 
(DAF; CD55), similar to PTEC line HK-2 and HEK293 cells, while this expression was lower on hESC-PEs (Fig. 1c).

Next, hESC-derived cells were tested for resistance or sensitivity to cytotoxic T cells. The hESC-PE was resistant to alloreactive CTLs directed against the specific HLA expressed by the $\mathrm{nESC}-\mathrm{PE}$ in a $4 \mathrm{~h}$ chromium release assay $(p>0.05)$, but became vulnerable to the CTLs after exposure to IFN $\gamma$, which had upregulated HLA ( $p=0.0005$; Fig. 2).

To test recognition by memory autoreactive CTLs, HLA matching had to be introduced. Beta cell-specific autoreactive memory CTLs of type 1 diabetes patients pose a particular threat to transplanted beta cells if transplanted beta cells match recipient HLA. HLA-A2 was lentivirally introduced in hESCECs under the constitutive EF1 $\alpha$ promoter $(55 \%$ transduction efficiency). It was thus found that hESC-ECs expressing insulin and HLA-A2 were specifically killed by PPI-specific CTLs with $>90 \%$ efficiency when compared with nonspecific CTLs $(p<0.0001$; Fig. 3$)$. The viability of mocktransduced insulin-expressing hESC-ECs incubated with PPI CTLs was comparable with that of transduced cells incubated with non-specific CTLs (73.2 and 71.5\%, respectively). Adding PPI-positive control peptide did not further increase killing of insulin-expressing cells, but did lead to recognition and killing of HLA-A2 transduced cells that did not express insulin (data not shown).

To confirm peptide-specific killing of hESC-derived cells through their cognate HLA, we assessed killing by CTLs recognising CMV peptide in cognate HLA-A1. Similar to alloreactive CTLs, CMV-specific CTLs did not effectively kill hESC-PEs loaded with CMV peptide unless the hESC-PEs were exposed to IFN $\gamma$ or the assay was prolonged. The differentiated hESC-ECs were vulnerable to alloreactive and CMV-specific CTLs without prior IFN $\gamma$ treatment, while prolonged exposure increased killing to $>90 \%$ (Fig. 2).

Alloreactive antibodies can lead to rejection of transplants through the induction of ADCC or activation of the complement cascade. Human antibodies specific for the HLA of the hESC-derived cells or a third party were used to induce cytotoxicity by peripheral blood lymphocytes or by rabbit complement. hESC-PEs were not sensitive to alloreactive antibodyinduced cytotoxicity unless HLA was upregulated by IFN $\gamma$ (Fig. 4). In vivo-differentiated hESC-ECs exhibited an increased sensitivity to antibody-induced cellular cytotoxicity without prior inflammatory HLA upregulation, but not to complement-dependent cytotoxicity, regardless of IFN $\gamma$ upregulation of HLA (Fig. 4).

\section{Discussion}

Insights into the immunogenicity of beta cell-like cells from sources that are alternatives to human cadaver islets will help the decision-making process when choosing immune-protective strategies to test and the most relevant immune monitoring to perform in clinical translation. hESC-PEs are promising candidates for clinical application [30]. We therefore investigated the immunological properties of hESC-derived pancreatic cell preparations before and after differentiation to endocrine cells. We found that hESC-PEs maintain immune privilege with low HLA expression, although upregulation appears possible in inflammatory conditions which then render the cells vulnerable to adaptive immune responses. In vivo differentiation of $h E S C$-PEs into a preparation with pancreatic endocrine cells also leads to immunological differentiation with increasing HLA expression and sensitivity to adaptive immune responses and also increased resistance to complement-mediated attack. Recognition and destruction by autoreactive PPI-specific T cells verified mature beta cell immune presentation by hESC-ECs.

Low expression of HLA by hESCs and hESC-derived cells underlies the hypoimmunogenicity observed in most published studies, effectively hiding cells from $\mathrm{T}$ cell recognition and alloreactive antibodies, while differentiation can upregulate HLA and increase vulnerability to immunity [17-21]. In our study, immunogenicity to alloreactive and peptide (CMV)-specific responses was related to HLA expression. hESC-PEs expressed very low levels of HLA in a noninflamed environment, making them hypoimmunogenic, while differentiation to hESC-ECs resulted in an increase in HLA expression and immunogenicity to T cells to levels reported for human islets in the literature [23, 31-34].

Despite the hypoimmunogenicity of hESC-PEs, resistance was incomplete after prolonged exposure to high numbers of CTLs. This suggests that hESC-PEs do not employ active suppression of CTL responses, as has been observed in other studies [21]. Further, HLA expression could be induced by mimicking inflammation with IFN $\gamma$. While this increased immunogenicity to alloreactive $\mathrm{T}$ cells and antibodies, the incomplete immune privilege may help to ensure adequate virus control in case of infection and prevent destruction by inflammation-activated NK cells [35].

Increased expression of HLA through inflammation or differentiation of hESC to beta cells may make cells vulnerable to recurrent autoimmunity if cells are transplanted to an HLAmatched individual with type 1 diabetes. After realising HLA match through transduction of in vivo-differentiated hESCECs, the insulin-expressing cells could be selectively eliminated by autoreactive CTLs previously isolated from a donor with type 1 diabetes [23]. Thus, we show hESC-derived beta cells risk recurrent autoimmunity in an HLA-matched setting. Further, by proving presentation of the PPI peptide recognised by the CTL clone, we verify that hESC-ECs have active cellular mechanisms in place to ensure immune surveillance and that they display genuine insulin production allowing natural presentation of PPI epitopes in HLA.

In vivo differentiation of hESC-PEs to hESC-ECs increased resistance to complement-mediated attack despite 
increased expression of antigen (HLA) recognised by alloreactive antibodies. Protection from complement is mediated through complement inhibitory receptors, such as CD46 (MCP), CD55 (DAF) and CD59 (MIP), which are known to be expressed by human islets [36,37]. All three receptors were expressed by the hESC-derived cells but CD55 expression was low on hESC-PEs, which may explain their vulnerability to complement-mediated cytotoxicity.

Although we were able to study diverse immune mechanisms relevant for transplantation of alternative beta cells, extrapolation of these results to the clinical transplantation setting is limited by several factors. First, animal models for in vivo differentiation of hESC-PEs to hESC-ECs may not completely reflect the differentiation process in humans. Second, culture conditions that are required for investigation of explanted in vivo-differentiated cells may alter the properties of the cells or the composition of the population. To this end, staining for beta cells did not show selective reduction of beta cells. Incomplete dissociation of naturally aggregating hESC-derived pancreatic endodermal cells and endocrine cells required for immune assays may contribute to the variation and relatively large standard error in the cytotoxicity assays (Figs 2, 4).

A head-to-head comparison with primary human islet cells would be of interest and was considered when setting up this study. However, it proved to be logistically impossible. hESCs require 4 months of in vivo differentiation into endocrine cells and the ex vivo experiments must be started within $24 \mathrm{~h}$ after explantation to assure optimal cell quality. The supply of fresh human islets for research purposes is highly infrequent and rare, precluding parallel testing with differentiated hESCECs. This was limited further by the HLA restrictions enforced by this type of experiment. We therefore looked to data on beta cell immunogenicity from the literature for the purpose of comparison $[23,31-34,36]$.

The identified immunogenicity of hESC-derived pancreatic endocrine cells highlights the need for an immune suppressive strategy for human transplantation. The vulnerability of these hESC-derived cells to cellular attack and their resistance to complement-mediated attack suggests that providing protection by cell-impermeable macro-encapsulation could be a successful strategy in clinical implementation. Alternatively, or additionally, the initial hypoimmunogenicity of the engrafted pancreatic endoderm may provide a window of opportunity in which to induce graft-specific tolerance.

In conclusion, pancreatic endoderm progenitors maintain hypoimmunogenicity, while differentiation of hESCs to pancreatic endocrine cells increases vulnerability to cellular immunity. Inflammatory conditions further increase immunogenicity. Yet, maturation of pancreatic endoderm to endocrine cells enforces resistance to complement-mediated cytotoxicity. This implies that, while simply preventing inflammation around transplantation of pancreatic endoderm may suffice initially, the maturing graft may need protection from cellular immune attack. Therefore, protecting grafts by cellimpermeable macro-encapsulation could be a successful strategy in clinical implementation. Alternatively, or in addition, the hypoimmunogenicity of the initially engrafted pancreatic endoderm may create a window of opportunity in which to induce graft-specific tolerance.

Acknowledgements The authors thank the European Commission and the JDRF for supporting this work and thank R. J. Lebbink (Medical Microbiology, University Medical Center Utrecht, Utrecht, the Netherlands) for the HLA-A2 expression vector and A. Mulder (IHB, LUMC) for providing human HLA specific antibodies.

Funding This work was funded by the European Commission (BetaCellTherapy, no. 241883 in the FP7 program) and the JDRF (172013-296).

Duality of interest LM, EK and EPB are employees of ViaCyte, receiving salary and stock options. DP is a member of ViaCyte's scientific and clinical advisory board. All other authors declare that there is no duality of interest associated with their contribution to this article.

Contribution statement The study was conceived and designed by CRT, DP and BOR, with MP, LM, EK and EPB also being responsible for the design. AZ, MP, GS, LM, EK, EPB and DP provided study material. CRT, AZ, GD, SHB-S and GS were responsible for collection and/or assembly of data and data analysis and interpretation. BOR was responsible for analysis and interpretation of data. CRT, AZ, LM, EK, EPB, DP and BOR wrote the manuscript and GD, SHB-S, MP and DP revised it. All authors approved the final version of the manuscript. BOR supervised the study and is the guarantor of this work.

Open Access This article is distributed under the terms of the Creative Commons Attribution 4.0 International License (http:// creativecommons.org/licenses/by/4.0/), which permits unrestricted use, distribution, and reproduction in any medium, provided you give appropriate credit to the original author(s) and the source, provide a link to the Creative Commons license, and indicate if changes were made.

\section{References}

1. Kroon E, Martinson LA, Kadoya K et al (2008) Pancreatic endoderm derived from human embryonic stem cells generates glucoseresponsive insulin-secreting cells in vivo. Nat Biotechnol 26:443452

2. Ravassard P, Hazhouz Y, Pechberty S et al (2011) A genetically engineered human pancreatic beta cell line exhibiting glucoseinducible insulin secretion. J Clin Invest 121:3589-3597

3. Shahjalal HM, Shiraki N, Sakano D et al (2014) Generation of insulin-producing $\beta$-like cells from human iPS cells in a defined and completely xeno-free culture system. J Mol Cell Biol 6:394408

4. Newby BN, Terada N, Mathews CE (2014) In search of a surrogate: engineering human beta cell lines for therapy. Trends Endocrinol Metab 25:378-380

5. Elliott RB, Living Cell T (2011) Towards xenotransplantation of pig islets in the clinic. Curr Opin Organ Transplant 16:195-200

6. Hering BJ, Cooper DK, Cozzi E et al (2009) The International Xenotransplantation Association consensus statement on 
conditions for undertaking clinical trials of porcine islet products in type 1 diabetes- executive summary. Xenotransplantation 16:196202

7. Schulz TC, Young HY, Agulnick AD et al (2012) A scalable system for production of functional pancreatic progenitors from human embryonic stem cells. PLoS One 7:e37004

8. Kirk K, Hao E, Lahmy R, Itkin-Ansari P (2014) Human embryonic stem cell derived islet progenitors mature inside an encapsulation device without evidence of increased biomass or cell escape. Stem Cell Res 12:807-814

9. Huurman VA, Hilbrands R, Pinkse GG et al (2008) Cellular islet autoimmunity associates with clinical outcome of islet cell transplantation. PLoS One 3:e2435

10. Roelen DL, Huurman VA, Hilbrands R et al (2009) Relevance of cytotoxic alloreactivity under different immunosuppressive regimens in clinical islet cell transplantation. Clin Exp Immunol 156: $141-148$

11. Qi M, Kinzer K, Danielson KK et al (2014) Five-year follow-up of patients with type 1 diabetes transplanted with allogeneic islets: the UIC experience. Acta Diabetol 51:833-843

12. Patel R, Terasaki PI (1969) Significance of the positive crossmatch test in kidney transplantation. N Engl J Med 280:735-739

13. Campbell PM, Salam A, Ryan EA et al (2007) Pretransplant HLA antibodies are associated with reduced graft survival after clinical islet transplantation. Am J Transplant 7:1242-1248

14. Hilbrands R, Gillard P, Van der Torren CR et al (2013) Predictive factors of allosensitization after immunosuppressant withdrawal in recipients of long-term cultured islet cell grafts. Transplantation 96: $162-169$

15. Raulet DH, Vance RE (2006) Self-tolerance of natural killer cells. Nat Rev Immunol 6:520-531

16. van der Torren CR, Zaldumbide A, Roelen DL et al (2016) Innate and adaptive immunity to human beta cell lines: implications for beta cell therapy. Diabetologia 59:170-175

17. English K, Wood KJ (2011) Immunogenicity of embryonic stem cell-derived progenitors after transplantation. Curr Opin Organ Transplant 16:90-95

18. Drukker M, Katz G, Urbach A et al (2002) Characterization of the expression of MHC proteins in human embryonic stem cells. Proc Natl Acad Sci U S A 99:9864-9869

19. Li L, Baroja ML, Majumdar A et al (2004) Human embryonic stem cells possess immune-privileged properties. Stem Cells 22:448-456

20. Robertson NJ, Brook FA, Gardner RL, Cobbold SP, Waldmann H, Fairchild PJ (2007) Embryonic stem cell-derived tissues are immunogenic but their inherent immune privilege promotes the induction of tolerance. Proc Natl Acad Sci U S A 104:20920-20925

21. Yachimovich-Cohen N, Even-Ram S, Shufaro Y, Rachmilewitz J, Reubinoff B (2010) Human embryonic stem cells suppress T cell responses via arginase I-dependent mechanism. J Immunol 184: $1300-1308$

22. Motte E, Szepessy E, Suenens K et al (2014) Composition and function of macroencapsulated human embryonic stem cellderived implants: comparison with clinical human islet cell grafts. Am J Physiol Endocrinol Metab 307:E838-E846
23. Skowera A, Ellis RJ, Varela-Calvino R et al (2008) CTLs are targeted to kill beta cells in patients with type 1 diabetes through recognition of a glucose-regulated preproinsulin epitope. J Clin Invest 118:3390-3402

24. Borst J, de Vries E, Spits H, de Vries JE, Boylston AW, Matthews EA (1987) Complexity of T cell receptor recognition sites for defined alloantigens. J Immunol 139:1952-1959

25. Amir AL, D Orsogna LJ, Roelen DL et al (2010) Allo-HLA reactivity of virus-specific memory $\mathrm{T}$ cells is common. Blood 115: 3146-3157

26. Mulder A, Kardol M, Blom J, Jolley WB, Melief CJ, Bruning JW (1993) Characterization of two human monoclonal antibodies reactive with HLA-B12 and HLA-B60, respectively, raised by in vitro secondary immunization of peripheral blood lymphocytes. Hum Immunol 36:186-192

27. Roep BO, Arden SD, De Vries RR, Hutton JC (1990) T cell clones from a type-1 diabetes patient respond to insulin secretory granule proteins. Nature 345:632-634

28. Carlotti F, Bazuine M, Kekarainen T et al (2004) Lentiviral vectors efficiently transduce quiescent mature 3T3-L1 adipocytes. Mol Ther 9:209-217

29. Hopkins KA (1990) Basic microlymphocytotoxicity test. In: Zachary AA, Terisi GA (eds) ASHI laboratory manual, 2nd edn. American Society of Histocompatibility and Immunogenetics, Lenexa, pp 49-80

30. Bruni A, Gala-Lopez B, Pepper AR, Abualhassan NS, Shapiro AJ (2014) Islet cell transplantation for the treatment of type 1 diabetes: recent advances and future challenges. Diabetes Metab Syndr Obes 7:211-223

31. Pujol-Borrell R, Todd I, Doshi M, Gray D, Feldmann M, Bottazzo GF (1986) Differential expression and regulation of MHC products in the endocrine and exocrine cells of the human pancreas. Clin Exp Immunol 65:128-139

32. Campbell IL, Bizilj K, Colman PG, Tuch BE, Harrison LC (1986) Interferon- $\gamma$ induces the expression of HLA-A, B, C but not HLADR on human pancreatic $\beta$-cells. J Clin Endocrinol Metab 62: 1101-1109

33. Campbell PD, Estella E, Dudek NL et al (2008) Cytotoxic Tlymphocyte-mediated killing of human pancreatic islet cells in vitro. Hum Immunol 69:543-551

34. Knight RR, Kronenberg D, Zhao M et al (2013) Human $\beta$-cell killing by autoreactive preproinsulin-specific CD8 T cells is predominantly granule-mediated with the potency dependent upon $\mathrm{T}$ cell receptor avidity. Diabetes 62:205-213

35. Pahl JH, Ruslan SE, Buddingh EP et al (2012) Anti-EGFR antibody cetuximab enhances the cytolytic activity of natural killer cells toward osteosarcoma. Clin Cancer Res 18:432-441

36. Bennet W, Bjorkland A, Sundberg B et al (2001) Expression of complement regulatory proteins on islets of Langerhans: a comparison between human islets and islets isolated from normal and hDAF transgenic pigs. Transplantation 72:312-319

37. Tai JH, Sun H, Liu W, Melling CW, Hasilo C, White DJ (2008) Isolating human islets of Langerhans causes loss of decay accelerating factor (CD55) on beta-cells. Cell Transplant 17:1349-1359 